\title{
Association of renal cyst and type A acute aortic dissection with hypertension
}

\author{
Jinlan Bao ${ }^{1 \#}$, Shaoxin Zheng ${ }^{1 \#}$, Canxia Huang ${ }^{2}$, Jun Tao ${ }^{3}$, Ying Tang ${ }^{1}$, Runlu Sun ${ }^{1}$, Qi Guo ${ }^{1}$, Jingfeng Wang ${ }^{1}$, \\ Yuling Zhang ${ }^{1}$
}

${ }^{1}$ Department of Cardiology, Sun Yat-sen Memorial Hospital, Sun Yat-sen University, Guangzhou, China; ${ }^{2}$ Department of Intensive Care Unit, Sun Yat-sen Memorial Hospital, Sun Yat-sen University, Guangzhou, China; ${ }^{3}$ Department of Cardiovascular Surgery, Sun Yat-sen Memorial Hospital, Sun Yat-sen University, Guangzhou, China

Contributions: (I) Conception and design: J Bao, S Zheng; (II) Administrative support: Y Zhang, J Wang; (III) Provision of study materials or patients: C Huang, J Tao; (IV) Collection and assembly of data: J Bao, S Zheng, Y Tang, R Sun; (V) Data analysis and interpretation: J Bao, S Zheng, C Huang, Q Guo; (VI) Manuscript writing: All authors; (VII) Final approval of manuscript: All authors.

\#These authors contributed equally to this work.

Correspondence to: Yuling Zhang; Jingfeng Wang. Sun Yat-sen Memorial Hospital, Sun Yat-sen University, No. 107, West Yanjiang Road, Guangzhou 510120, China. Email: zhyul@mail.sysu.edu.cn; wjingf@mail.sysu.edu.cn.

Background: Type A acute aortic dissection (TA-AAD) has high mortality, with $50 \%$ of patients dying before hospital admission. Hypertension is the most common comorbidity for acute aortic dissection, and effective antihypertensive therapy is still unable to predict the risk of aortic rupture at the medium- and longterm stages. While the presence of renal cyst has been found to increases the risk of thoracic aortic disease, the correlation between renal cyst and TA-AAD with hypertension remains poorly understood. Thus, this study aimed to determine the relationship of renal cyst and TA-AAD with hypertension.

Methods: A retrospective analysis was performed in 464 hypertension patients from August 2014 to August 2019. A total of $230 \mathrm{TA}-\mathrm{AAD}$ patients were enrolled in the $\mathrm{AD}$ with hypertension group (age $53.79 \pm 11.31$ years, male $90.87 \%$ ), and matched by age, sex, and hypertension control to 234 patients without TA-AAD who were enrolled in the non-AD with hypertension group. Patients were divided into three subgroups according to the numbers of renal cysts: no renal cyst, single renal cyst, and multiple renal cysts.

Results: In this study, the $\mathrm{AD}$ with hypertension group had significantly more single renal cyst and multiple renal cyst cases than did the non-AD with hypertension group. The mean age of the multiple renal cyst subgroup was significantly older than that of the single renal cyst subgroup $(57.25 \pm 13.00 v$ s. $51.57 \pm 10.75$ years $)$ in the $\mathrm{AD}$ with hypertension group. There was significantly different distribution of dissection starting points and dissection ending points across three renal cyst subgroups. Multivariate logistic regression analysis indicated that having no renal cyst significantly decreased the risk of TA-AAD in middle-aged and elderly patents, but showed no correlations with those of younger ages. Single renal cyst status also significantly decreased the risk of TA-AAD in elderly patients [odds ratio $(\mathrm{OR})=0.129,95 \%$ confidence interval (CI): 0.029-0.575, P=0.007].

Conclusions: Renal cyst status correlates with the risk of TA-AAD with hypertension in middle-aged and elderly patients, and exhibits different degrees of vascular lesion in aortic dissection. We therefore suggest that different antihypertensive standards should be adopted in different renal cyst status to more effectively prevent aortic dissection.

Keywords: Type A acute aortic dissection (TA-AAD); hypertension; renal cyst

Submitted Nov 18, 2020. Accepted for publication Dec 16, 2020.

doi: $10.21037 /$ jtd-20-3422

View this article at: http://dx.doi.org/10.21037/jtd-20-3422 


\section{Introduction}

Type A acute aortic dissection (TA-AAD) is mainly performed by aortic dissection or rupture, and about $50 \%$ of patients who undergo this procedure die before hospital admission (1-3). Thoracic aortic dissection includes Stanford type A and type B, type A is involved in the ascending aorta, but Type $\mathrm{B}$ indicates that the dissection is limited to the descending aorta (4). Despite the advancements in diagnostic imaging methods and surgical techniques, the mortality of TA-AAD ranges from $14 \%$ to $30 \%$ with a $25 \%$ average mortality rate (5-7). A recent analysis follow-up 30,412 middle-aged patients (mean age $58.0 \pm 7.6$ years) for 20 years reported that an aortic dissection incidence of 15 per 100,000 patientyears (8), with this condition typically being clinically silent until potentially fatal complications of dissection or rupture develop. The average ages of patients presenting with acute aortic dissection have been reported to range from 48 to 67 years (median age 61 ) with $50 \%$ to $81 \%$ being men $(8,9)$. Recently, several studies have confirmed that uncontrolled hypertension is a significant treatable risk factor for acute aortic dissection $(1,10,11)$. In observational studies, $65-75 \%$ TA-AAD with hypertension (11), found that hypertension was the most common comorbidity for acute aortic dissection, with almost $45 \%$ to $100 \%$ of these patients having hypertension (9). However, long-term antihypertensive therapy, there are still a lot of aortic dissection and even sudden death, so hypertension is unable to predict the risk of aortic rupture at the medium- and long-term stages.

There is a considerable amount of research which indicates that maximal aortic diameter can predict aortic dissection with hypertension, but this issue nonetheless remains controversial. Kim et al. found maximal aortic diameter at baseline to be an exclusively significant predictor of aortic events, and estimated the probability of aortic events occurring within 1 year to be $5.5 \%, 7.2 \%$, and $9.3 \%$ for aortic diameters of 50,55 , and $60 \mathrm{~mm}$, respectively (12); in contrast, Pape et al. reported that aortic dissection occurred in up to $60 \%$ of individuals with an ascending aortic aneurysm size $<5.5 \mathrm{~cm}$, and that an aortic diameter $>5.5 \mathrm{~cm}$ is not a good predictor for TA-AAD $(6,13)$. A few novel biomarkers for acute aortic dissection, such as soluble ST2 and D-Dimer have been discovered; however, their effectiveness remains controversial (14-16). Some indicators, such as vascular endothelial growth factor pathway inhibitors (VPIs), rcan1, small GTP-binding protein, and vascular smooth muscle cells have been found to be capable of predicting thoracic or abdominal aortic aneurysm (AAA) formation and rupture, but little research was been conducted on TA-AAD in this regard (17-20). Body mass index was demonstrated to have a negative linear association with female aortic dissection AD mortality, while trends in systolic blood pressure have exhibited a positive linear relationship with male AD mortality (21). Due to the lack of clinical research, however, the above measures cannot be widely applied in clinical practice.

Simple renal cyst (SRC) can likely be used as a marker for the timely detection of patients at risk of thoracic aortic disease (22). The study included ascending aortic aneurysm, descending aortic aneurysm, type B aortic dissection and type A aortic dissection, of which only 118 cases of type A aortic dissection, mainly studies the correlation between single renal cyst and the prevalence of four subgroup. Whether renal cyst was associated with the severity of aortic dissection was not discussed. In Kim's study, a common manifestation of connective tissue degeneration from 518 patients with aortic dissection and 1,366 healthy subjects indicated that the presence of renal cyst is associated with increased risk of aortic dissection (23). A pooled analysis of 7 estimates from the five studies demonstrated a statistically significant 2.54-fold prevalence of SRC in patients with AAA relative to subjects without AAA [odds ratio (OR), 2.54; 95\% confidence interval (CI), 1.93-3.34; $\mathrm{P}<0.00001]$ (24). Of a total of 35,498 patients, 6,366 were found to have SRC. Compared with the matched population without SRC, individuals with SRC were significantly more likely to experience type A aortic dissection ( $0.6 \%$ vs. $0.2 \%)$ (13). However, the association between renal cyst and TA-AAD with hypertension remains unknown.

The aim of this study was thus to determine the relationship between renal cyst and TA-AAD with hypertension.

We present the following article in accordance with the STROBE reporting checklist (available at http://dx.doi. org/10.21037/jtd-20-3422).

\section{Methods}

\section{Study population}

An $\mathrm{AD}$ with hypertension group was assigned, comprising 230 hypertension patients who were diagnosed as TAAAD by computed tomography angiography (CTA) and who accepted surgical aortic replacement. The non-AD with hypertension group comprised 234 hypertension patients in whom TA-AAD was excluded by CTA. The two 
groups were completely matched according to age, sex, and hypertension control. According to the numbers of renal cysts, they were further divided into three subgroups: no renal cyst, single renal cyst, and multiple renal cysts (number of cysts $\geq 2)$. There were also three age distributions: young ( $\leq 39$ years), middle-aged ( $40-59$ years), and elderly ( $\geq 60$ years). All participants were selected from the Sun Yatsen Memorial Hospital, Sun Yat-sen University in China, from August 2014 to August 2019. Patients with Marfan syndrome, pregnancy, autosomal dominant polycystic kidney disease (ADPKD), end-stage renal disease, and hydronephrosis were excluded. This study was approved by the human investigation committee of Sun Yat-sen Memorial Hospital and was conformed to the provisions of the Declaration of Helsinki (as revised in 2013). Because of the retrospective nature of the research, the requirement for informed consent was waived.

\section{Determining the data of echocardiogram and renal cyst}

We collected ultrasonic cardiogram data which included information of the ascending aorta (AAO), ventricular septum dimension (IVSD), left atrium (LA), left ventricular end diastolic dimension (LVDD), left ventricular posteriorwall dimension (LVPWD), and the left ventricular ejection fraction (LVEF). We recorded the number of renal cysts using renal ultrasonic examination, with renal cysts being defined as a thin-walled, low-attenuation, oval-to-round lesions with a diameter $\geq 4 \mathrm{~mm}$. According to the number of renal cysts, a patient could be delineated into a non-renal cyst, single renal cyst, or multiple renal cyst (number of cysts $\geq 2$ ) subgroup.

\section{Radiology imaging manifestations in TA-AAD}

Thoracic aortic dissection includes Stanford type (A and B) and DeBakey type (I-II) (4). DeBakey I: the dissection originates from the ascending aorta and extends beyond the aortic arch to the descending aorta and even to the abdominal aorta, this type is most common; DeBakey II: the dissection arises from the ascending aorta; DeBakey III: the dissection originates from the descending aorta and extends distal to the abdominal aorta (25). Stanford type $\mathrm{A}$ is involved in the ascending aorta, but Type B indicates that the dissection is limited to the descending aorta (Figure S1). So, Stanford A is equal to DeBakey I and DeBakey II. In all, 230 TA-AAD patients were confirmed by computed tomography (CT) imaging, DeBakey I patients 200
(86.96\%) and DeBakey II 30 (13.04\%). We then recorded the point where the dissection started and where it ended (which could include the ascending aortic, aortic arch, descending aortic, renal artery, mesenteric artery, and iliac artery).

\section{Other clinical and biochemical risk factors}

Aortic atherosclerosis increased the risk of aortic dissection (26). The following risk factors were evaluated in the study population and were defined as follows:

(I) Hypertension and good hypertension control = systolic blood pressure (SBP) $\geq 140 \mathrm{mmHg}$ and/ or diastolic blood pressure (DBP) $\geq 90 \mathrm{mmHg}$ or history of hypertension with antihypertension medicinal treatment; good control $=\mathrm{SBP}$ $<140 \mathrm{mmHg}$ and DBP <90 mmHg;

(II) Hyperlipidemia $=$ total cholesterol (TC) level $\geq 5.17 \mathrm{mmol} / \mathrm{L}$ and/or treatment with lipid control medicine therapy;

(III) Diabetes = history of type 1 and type 2 diabetes;

(IV) Smoking history = at least a 2-year history of smoking;

(V) Alcohol history = drinking $50 \mathrm{~g}$ of wine every day;

(VI) Cerebral infarction history $=$ a history of significantly pathological conditions affecting the central or peripheral nervous systems, as confirmed by cerebral CT;

(VII) Positive family history $=$ the presence of confirmed family history of aneurysm, aortic dissection, and/or hypertension;

(VIII) Previous cardiac disease $=$ a history of coronary heart disease (CHD) with medicine or surgery.

We also collected the related biochemical risk factors, such as TC, triglyceride (TG), high density lipoprotein cholesterol (HDL-C), low density lipoprotein cholesterol (LDL-C), apolipoprotein A1 (apoA1), apolipoprotein B (apoB), and fast blood glucose (FBG).

\section{Statistical analysis}

Continuous variables are presented as mean \pm standard deviation. An independent-samples $t$-test was used to compare continuous variables, and one-way ANOVA was used to compare multiple continuous variables. Categorical variables are presented as numbers (\%). Pearson's chisquare test was used to compare categorical variables. Multivariate logistic regression analysis was used to indicate the correlation between renal cyst and the risk of TA-AAD, 
with adjustments made for AAO, IVSD, LVDD, LVPWD, TC, and TG. A P value $<0.05$ was considered as statistically significant. All statistical analyses were performed by using SPSS 23.0 (IBM, Armonk, NY, USA).

\section{Results}

Clinical baseline characteristics in the AD with bypertension group and the non-AD with bypertension group

In this study, the AD with hypertension group had significantly more single renal cyst patients [79 (34.35\%) vs. $50(21.37 \%), \mathrm{P}<0.001]$ and multiple renal cyst patients [51 (22.17\%) vs. 20 (8.55\%), $\mathrm{P}<0.001]$ than the non-AD with hypertension group (Table 1). Also, AAO, IVSD, LVDD, LVPWD, TC, and TG levels were significantly higher in the $\mathrm{AD}$ with hypertension group than in the non-AD hypertension group $(\mathrm{P}<0.05)$. In the $\mathrm{AD}$ with hypertension group, female hypertension optimal control was significantly lower than that in the non-AD with hypertension group the $[14(66.67 \%)$ vs. 17 (73.91\%); $\mathrm{P}=0.039]$ (Table 2).

\section{Clinical baseline characteristics in the three subgroups}

Patients were further divided, according to the numbers of renal cysts into a non-renal cyst, single renal cyst, or multiple renal cyst (number of cysts $\geq 2$ ) subgroup. In the $\mathrm{AD}$ with hypertension group, the mean age of the multiple renal cyst subgroup was significantly greater than that of the single renal cyst subgroup $(57.25 \pm 13.00$ vs. $51.57 \pm 10.75$ years $)$ (Table 3).

\section{Clinical baseline characteristics in the three age distributions}

Aortic dissection occurred mainly in middle-aged and elderly patients, and no significant difference between male and female patients was observed. However, all patients in the younger age group were male. Mean age significantly differed across the three age distributions in both the AD with hypertension group and the non-AD with hypertension group $(\mathrm{P}<0.05 ;$ Table 4$)$.

\section{Radiology imaging manifestations in the AD with bypertension group}

For radiology imaging, we recorded the dissection starting point and ending point. The prevalence of DeBakey I/ DeBakey II classifications significantly differed across the three renal cyst subgroups $(\mathrm{P}=0.042)$, and the no renal cyst subgroup had significantly more DeBakey II cases than the single renal cyst subgroup [19 (9\%) vs. 5 (6.33\%), $\mathrm{P}=0.015]$, but no difference in age distribution was observed (Figure $1 A, B)$. The prevalence of dissection end point differed significantly across the three renal cyst subgroups $(\mathrm{P}=0.002)$, but no difference in age distribution was observed (Figure 2A,B).

\section{Relationship of renal cyst and TA-AAD with bypertension}

Multivariate logistic regression analysis indicated that having no renal cysts significantly decreased the risk of TA$\mathrm{AAD}$ in males (OR $=0.185,95 \% \mathrm{CI}$ : 0.090-0.381, $\mathrm{P}<0.001)$. Furthermore, AAO increased the risk of TA-AAD in both males and females (Table 5). In the younger age group, IVSD was found to increase the risk of TA-AAD, but renal cysts had no correlation with the risk of TA-AAD (Table 6). In the middle-aged and elderly patients, having no renal cyst significantly decreased the risk of TA-AAD, and having only a single renal cyst also significantly decreased the risk of TA-AAD in the elderly patients $(\mathrm{OR}=0.129,95 \% \mathrm{CI}$ : 0.029-0.575, $\mathrm{P}=0.007)$.

\section{Discussion}

TA-AAD is a high fatality disease. The underlying cause of aortic dissection is multifactorial, with hypertension being the most significant treatable risk factor for acute aortic dissection (10). However, hypertension is still unable to predict the risk of aortic rupture at an early stage. In this study, we aimed to explore whether renal cyst status correlated with TA-AAD with hypertension. We found that TA-AAD mainly occurred in middle-aged and elderly individuals, and a small proportion occurred in younger males, which is consistent with the findings of other studies $(8,9)$. In this study, the percentage of males accounted for more than $90.87 \%(n=209)$ of the patients, which is more than that reported in a previous study (50-81\%) (9). We included cases of hypertension combined with AD, which perhaps explains the higher proportion of males in this study. The prevalence of SRCs was $34.35 \%$ in the AD with hypertension group and $2.37 \%$ in the non-AD with hypertension group, which is a statistically significant difference $(\mathrm{P}<0.001)$, and in line with previous research $(23)$. The prevalence of multiple renal cysts was $22.17 \%$ in the 
Table 1 Comparison of baseline characteristics between the AD with hypertension and non-AD with hypertension groups

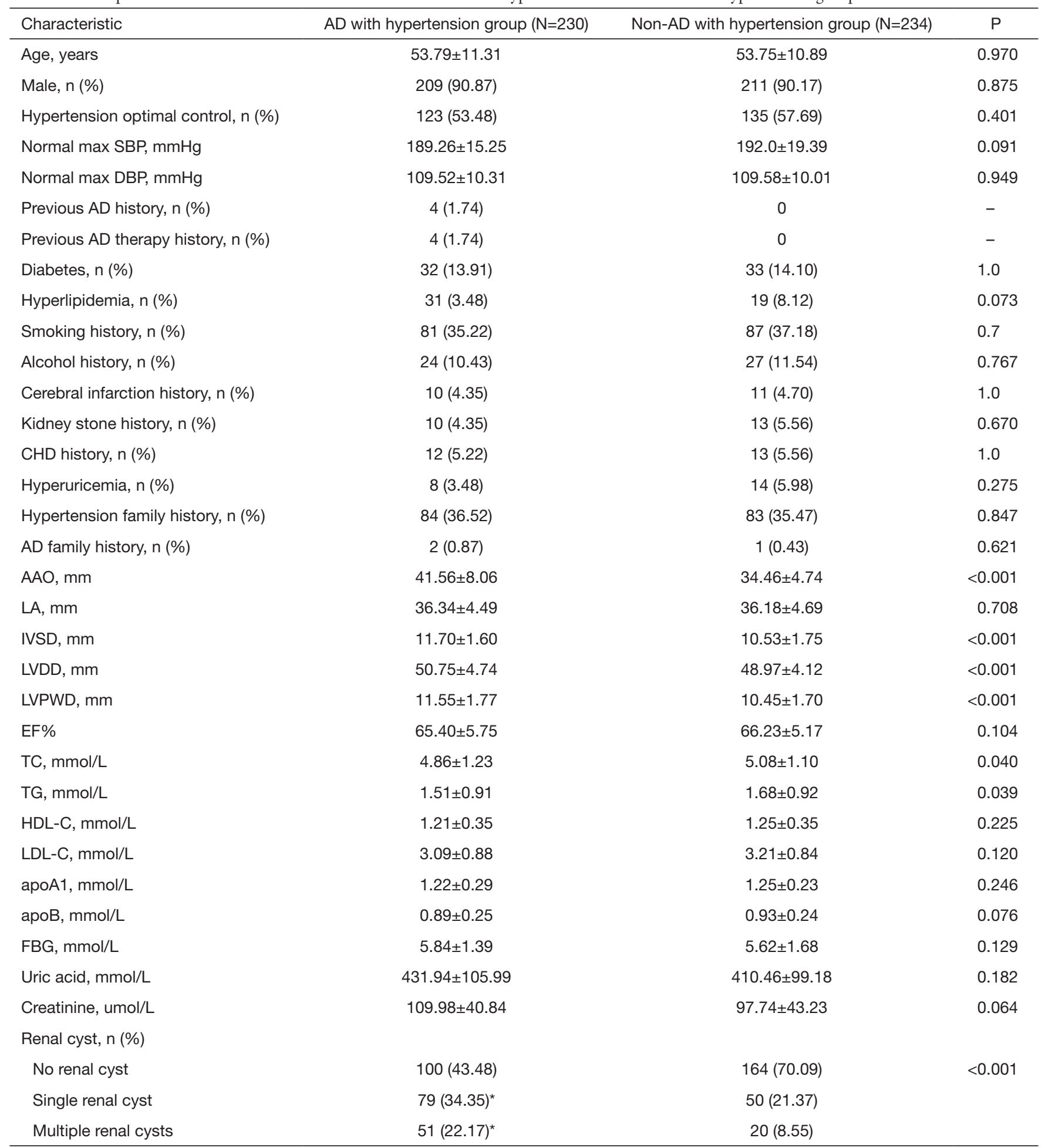

Renal cyst association with type $A$ acute aortic dissection with hypertension. *, compared with no renal cyst, $P<0.001 . A D$, aortic dissection; SBP, systolic blood pressure; DBP, diastolic blood pressure; CHD, coronary heart disease; AAO, ascending aorta; LA, left atrium; IVSD, ventricular septum dimension; LVDD, left ventricular end diastolic dimension; LVPWD, left ventricular posterior-wall dimension; EF, left ventricular ejection fraction; TC, total cholesterol; TG, triglyceride; HDL-C, high density lipoprotein cholesterol; LDL-C, low density lipoprotein cholesterol; FBG, fast blood glucose. 
Table 2 Comparison of baseline characteristics of males and females between the AD with hypertension and the non-AD with hypertension groups

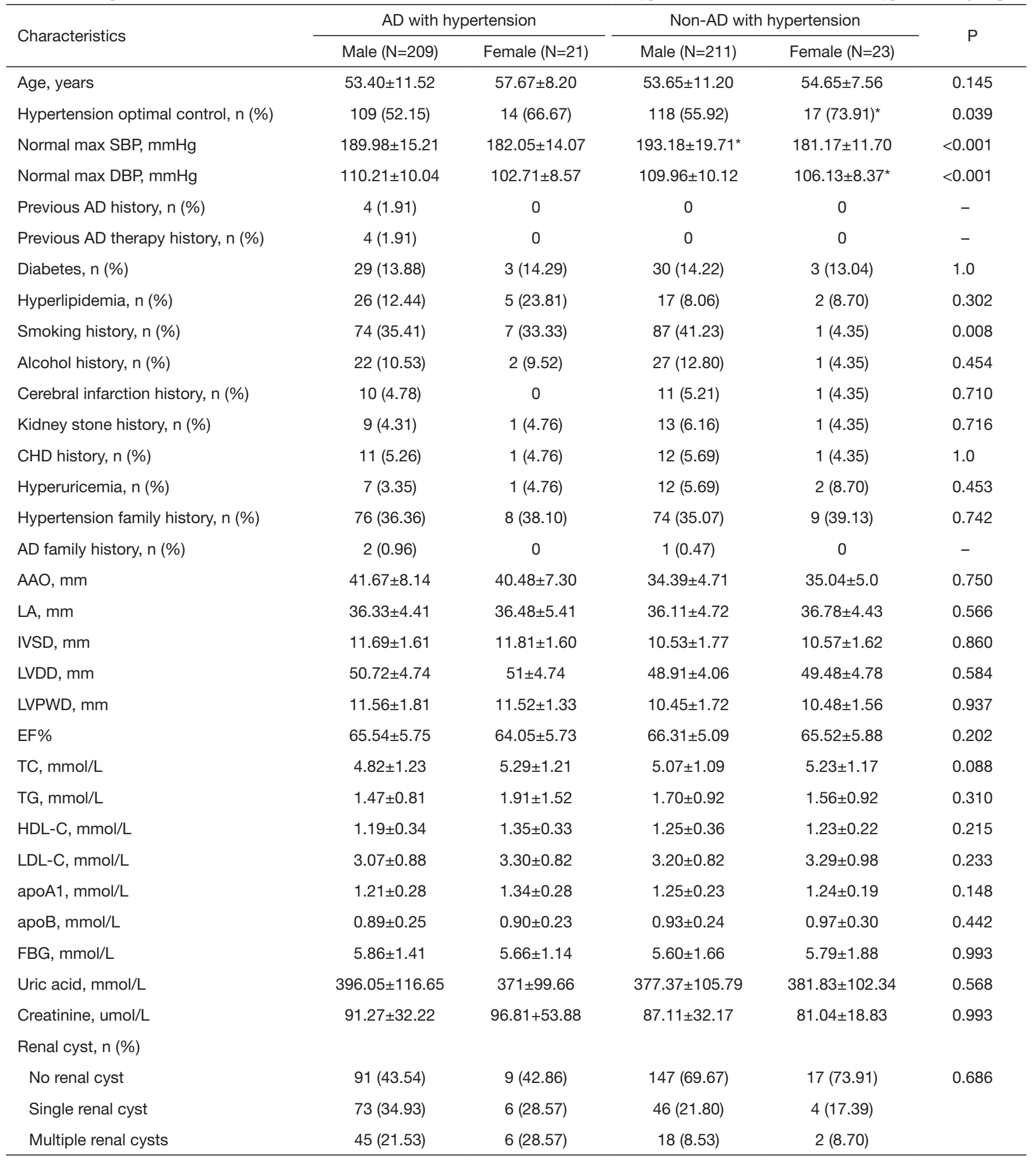

*, compared to $\mathrm{AD}$ with hypertension, $\mathrm{P}<0.05$. AD, aortic dissection; SBP, SBP, systolic blood pressure; DBP, diastolic blood pressure; $\mathrm{CHD}$, coronary heart disease; AAO, ascending aorta; LA, left atrium; IVSD, ventricular septum dimension; LVDD, left ventricular end diastolic dimension; LVPWD, left ventricular posterior-wall dimension; EF, left ventricular ejection fraction; TC, total cholesterol; TG, triglyceride ; HDL-C, high density lipoprotein cholesterol; LDL-C, low density lipoprotein cholesterol; FBG, fast blood glucose. 
Table 3 Comparison of baseline characteristics in the three renal cyst subgroups




Table 4 Comparison of baseline characteristics in the three age groups



*, compared with the younger age group, $\mathrm{P}<0.05$; ", compared with the elderly group, $\mathrm{P}<0.05$. AD, aortic dissection; SBP, systolic blood pressure; DBP, diastolic blood pressure; CHD, coronary heart disease; AAO, ascending aorta; LA, left atrium; IVSD, ventricular septum dimension; LVDD, left ventricular end diastolic dimension; LVPWD, left ventricular posterior-wall dimension; EF, left ventricular ejection fraction; TC, total cholesterol; TG, triglyceride ; HDL-C, high density lipoprotein cholesterol; LDL-C, low density lipoprotein cholesterol; FBG, fast blood glucose. 

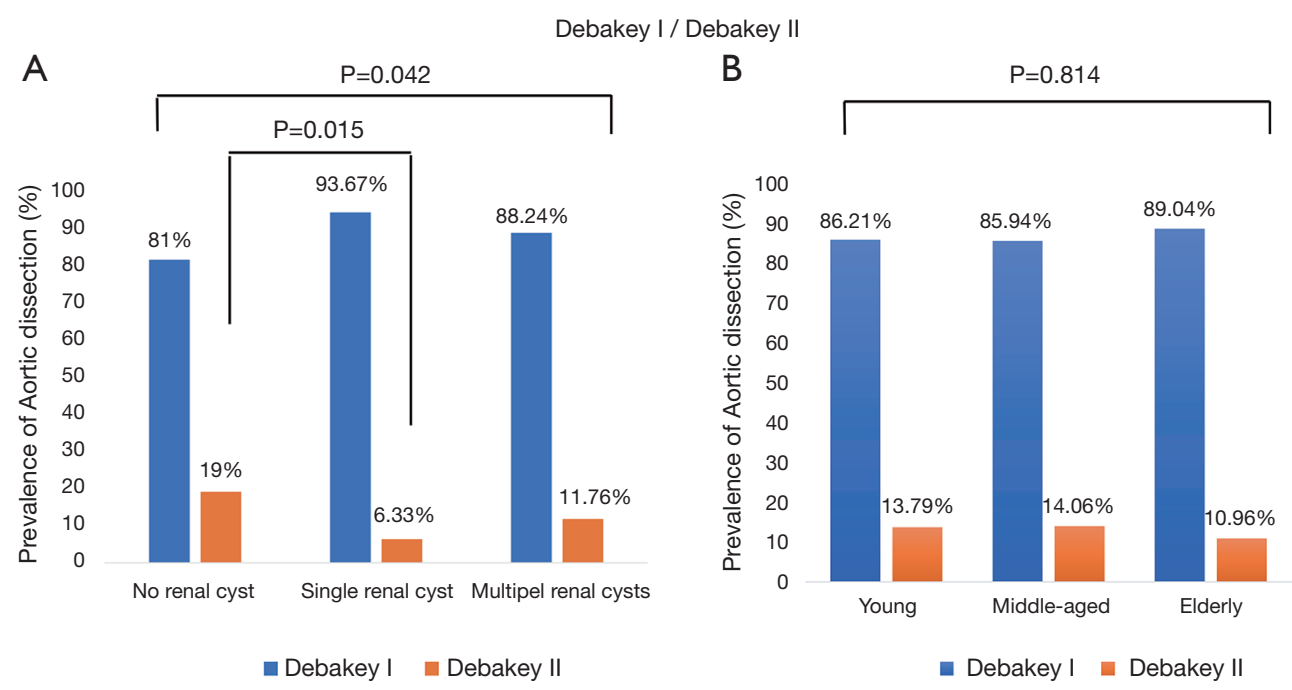

Figure 1 DeBakey I/DeBakey II classification for renal cyst and age subgroups. (A) The number of DeBakey I/DeBakey II cases differed across the three renal cyst subgroups $(\mathrm{P}=0.042)$, compare with the single renal cyst subgroup, no renal cyst subgroup having significantly more DeBakey II cases (19\% vs. 6.33\%, P=0.015). (B) There was no difference in DeBakey I/DeBakey II classification across the different age subgroups $(\mathrm{P}=0.814)$.

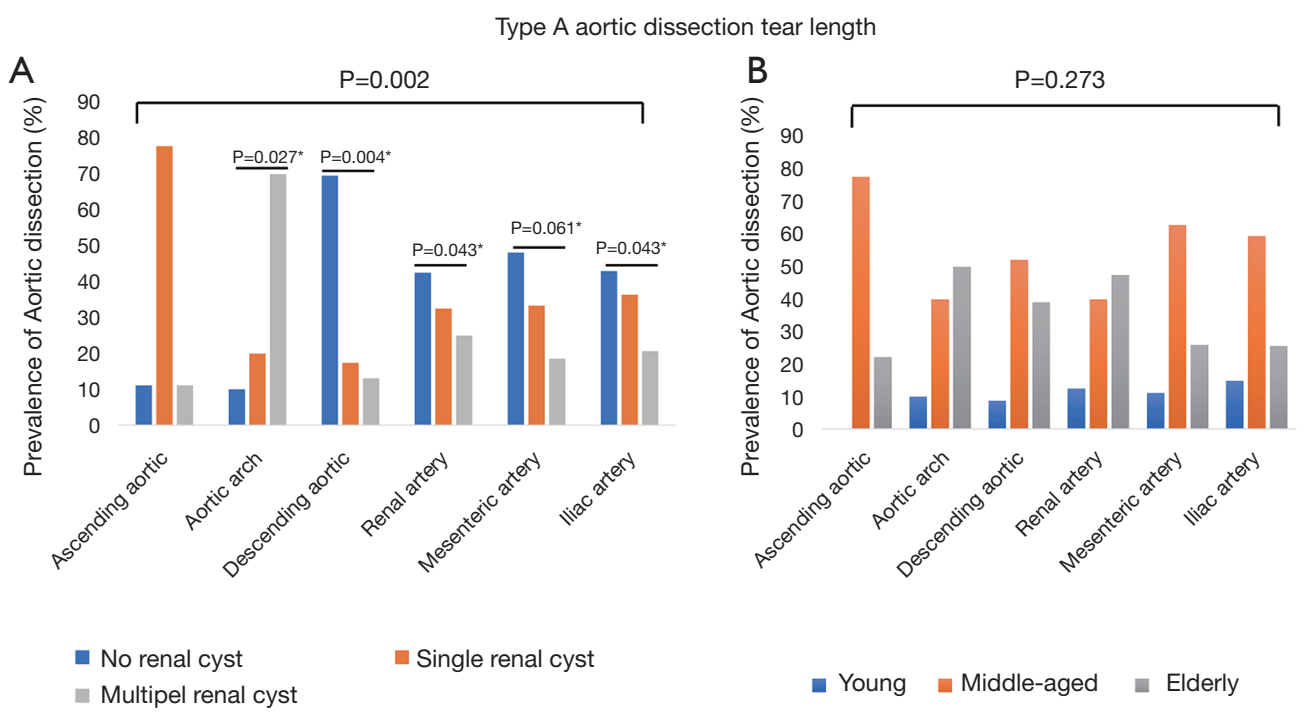

Figure 2 Type A aortic dissection tear length in the renal cyst and age subgroups. (A) The ending point differed across the three renal cyst subgroups ( $\mathrm{P}=0.002 ;$ *, significantly different compared with the ascending aorta); (B) there was no difference in dissection ending points across the three age subgroups $(\mathrm{P}=0.273)$.

$\mathrm{AD}$ with hypertension group which was significantly greater than the $8.55 \%$ in the non-AD with hypertension group $(\mathrm{P}<0.001)$; furthermore, patients with a single renal cyst in the $\mathrm{AD}$ with hypertension group tended to be older, which is consistent with the finding that the number of renal cysts increases with age (22).
Whether or not maximum aortic diameter is a predictor for aortic dissection is still a matter of controversy. Some studies have indicated that maximal diameter in the descending and abdominal aorta and older age are independent risk factors for rupture. The mere presence of uncharacteristic pain and a history of chronic obstructive 
Table 5 Multiple logistic regression analysis relationship between renal cyst and the risk of TA-AAD in different sex group

\begin{tabular}{|c|c|c|c|c|c|c|}
\hline Characteristics & \multicolumn{2}{|c|}{ All participants } & \multicolumn{2}{|l|}{ Male } & \multicolumn{2}{|l|}{ Female } \\
\hline AAO & $1.171(1.124,1.220)$ & $<0.001$ & $1.176(1.126,1.228)$ & $<0.001$ & $1.204(1.005,1.442)$ & 0.044 \\
\hline IVSD & $1.303(1.026,1.656)$ & 0.030 & $1.280(0.999,1.640)$ & 0.051 & $3.339(0.480,23.200)$ & 0.223 \\
\hline LVDD & $0.996(0.943,1.053)$ & 0.898 & $1.001(0.944,1.061)$ & 0.981 & $0.948(0.789,1.138)$ & 0.566 \\
\hline TC & $0.901(0.744,1.112)$ & 0.356 & $0.902(0.729,1.116)$ & 0.342 & $0.997(0.507,1.962)$ & 0.993 \\
\hline TG & $0.814(0.615,1.077)$ & 0.150 & $0.715(0.517,0.987)$ & 0.042 & $1.362(0.609,3.046)$ & 0.452 \\
\hline No renal cyst & $0.188(0.095,0.372)$ & $<0.001$ & $0.185(0.090,0.381)$ & $<0.001$ & $0.532(0.043,6.640)$ & 0.624 \\
\hline Single renal cyst & $0.448(0.214,0.939)$ & 0.033 & $0.454(0.209,0.986)$ & 0.046 & $0.967(0.056,16.805)$ & 0.982 \\
\hline
\end{tabular}

Adjusted for AAO, IVSD, LVDd, LVPWD, TC, TG. A P value $\leq 0.05$ was considered as statistically significant. TA-AAD, type A acute aortic dissection; AAO, ascending aorta; IVSD, ventricular septum dimension; LVDD, left ventricular end diastolic dimension; LVPWD, left ventricular posterior-wall dimension; TC, total cholesterol; TG, triglyceride.

Table 6 The relationship between renal cyst and the risk of TA-AAD in the three renal cyst subgroups according to multiple logistic regression analysis

\begin{tabular}{|c|c|c|c|c|c|c|}
\hline Characteristics & \multicolumn{2}{|l|}{ Young } & \multicolumn{2}{|c|}{ Middle-aged } & \multicolumn{2}{|l|}{ Elderly } \\
\hline AAO & $1.147(0.986,1.335)$ & 0.075 & $1.157(1.101,1.216)$ & $<0.001$ & $1.243(1.130,1.368)$ & $<0.001$ \\
\hline IVSD & $2.024(1.026,3.995)$ & 0.042 & $1.153(0.835,1.592)$ & 0.387 & $1.393(0.680,2.853)$ & 0.365 \\
\hline LVDD & $1.147(0.923,1.426)$ & 0.217 & $1.00(0.933,1.072)$ & 0.996 & $0.962(0.857,1.079)$ & 0.508 \\
\hline $\mathrm{TC}$ & $0.952(0.358,2.532)$ & 0.922 & $0.970(0.753,1.249)$ & 0.812 & $0.656(0.439,0.981)$ & 0.040 \\
\hline TG & $0.526(0.189,1.465)$ & 0.219 & $0.866(0.601,1.248)$ & 0.442 & $0.891(0.542,1.463)$ & 0.647 \\
\hline No renal cyst & $0.064(0.004,1.0)$ & 0.050 & $0.350(0.139,0.879)$ & 0.025 & $0.076(0.021,0.282)$ & $<0.001$ \\
\hline Single renal cyst & $0.098(0.006,1.628)$ & 0.105 & $0.987(0.366,2.660)$ & 0.980 & $0.129(0.029,0.575)$ & 0.007 \\
\hline
\end{tabular}

Adjusted for AAO, IVSD, LVDd, LVPWD, TC, TG. A P value $\leq 0.05$ was considered as statistically significant. TA-AAD, type A acute aortic dissection; AAO, ascending aorta; IVSD, ventricular septum dimension; LVDD, left ventricular end diastolic dimension; LVPWD, left ventricular posterior-wall dimension; TC, total cholesterol; TG, triglyceride.

pulmonary disease are considered as significant predictors of aortic events (27-31). In one study of 184 consecutive patients, including 108 surgically treated type $\mathrm{A} A D$ and 76 medically treated type $\mathrm{B} A \mathrm{D}$ who were discharged after an acute aortic dissection with patent false lumen, multivariate analysis identified baseline maximum descending aorta diameter as a predictor of dissection-related adverse events and mortality (31). Sueyoshi et al. examined 62 type B double-barrel aortic dissection patients with regular followup CT and found that the presence of blood flow in the false lumen was the only significant risk factor for increasing the diameter for aortic enlargement (28). In contrast, other studies have indicated that in the majority of patients with acute type A aortic dissection, neither aortic diameter $<5.5 \mathrm{~cm}$ nor aortic diameter $>5.5 \mathrm{~cm}$ were good predictors for type A aortic dissection; thus, methods other than size 
measurement of the ascending aorta are needed to further identify patients at risk for dissection (6). In this study, we found that the proportion of patients with AAO diameter $\geq 55 \mathrm{~mm}$ was $7.37 \%$, which is lower than that reported in Pape's study (6), but AAO nonetheless was found to increase the risk of TA-AAD in middle-aged and elderly patients in this study.

Previous research has also reported that the prevalence of SRC is highly correlated with the development of aortic dissection. In Kim's study, which included 518 patients with aortic dissection (AD group) and 1,366 healthy subjects who underwent CT for routine health screening (control group), the prevalence of SRC was significantly higher in the AD group than that the control group; additionally, multivariate analysis indicated that the presence of renal cyst could be a marker for predicting aortic dissection (23). Meanwhile, Ziganshin et al. evaluated the prevalence of SRC in 842 patients with thoracic aortic disease (TAD) and found that the prevalence of SRC was $44.1 \%$ for patients with type A dissection, while the prevalence of SRC in the control group was just $15.3 \%$, suggesting that increased prevalence of SRC in patients with TAD and SRC can potentially be used to predicted timely detection of patients at risk of TAD (22). One study assessed the prognostic value of SRC in type B aortic dissection (BAD) patients with hypertension $(n=238$, age $56.1 \pm 9.8$ years, $84.0 \%$ male) after thoracic endovascular aortic repair (TEVAR), with the results indicating that SRC could predict 24-month aortic-related adverse events in BAD patients with hypertension after TEVAR, especially in the chronic group (32). In our study, the mean age of the multiple renal cyst subgroup was significantly greater than that of the single renal cyst group in $\mathrm{AD}$ with hypertension patients. Having no renal cyst significantly decreased the risk of TA-AAD in middle-age and elderly patients, but no correlation was found with the younger age group. Having a single renal cyst also significantly decreased the risk of TA$\mathrm{AAD}$ in elderly patients. We can conclude that the number of renal cysts increases with age and becomes another important cause of TA-AAD with hypertension.

Early diagnosis of secondary dilatation of the diseased aorta is crucial to reducing mortality (33). Electrocardiography and chest $\mathrm{X}$-ray are often unable to confirm TA-AAD, and CT or magnetic resonance imaging (MRI) should not be delayed if TA-AAD is suspected (9,34-36). In one follow-up study of patients who underwent surgery for TA-AAD, a finding of a large false lumen with an area of the true lumen $<30 \%$ at 6 months after surgery was the strongest predictor for secondary dilatation of the diseased downstream aorta (33). In our study, dissection starting and ending points significantly different across the three renal cyst subgroups, and we thus can surmise that renal cyst status may correlate with different degrees of vascular lesion in aortic dissection. Consequently, for patients with hypertension and renal cyst, we suggest a lower blood pressure than normal control standards be maintained in order to more effectively prevent aortic dissection.

Some limitations in this study should also be addressed. These include a lack of follow-up and the inevitable preselection bias associated with retrospective analyses. Second, this study used a small sample from a single center, and thus the results cannot be generalized to the general population or other ethnicities.

\section{Conclusions}

Renal cysts significantly increase the risk of TA-AAD with hypertension in middle-aged and elderly patients, and different renal cysts lead to varying degrees of aortic vascular disease. We therefore recommend that for patients with hypertension, different antihypertensive standards should be adopted corresponding to the specific renal cyst status in order to more effectively prevent aortic dissection.

\section{Acknowledgments}

The authors would like to thank professors Yiming Zhou, Yue Pan, and Zhaoyu Liu (all from Sun Yat-sen Memorial Hospital, Sun Yat-sen University, China) for their guidance in the project design.

Funding: This study received funding from the Guangzhou Science and Technology Fund (No. 201803040010), the National Natural Science Foundation of China (No. 8197021630), the Foundation and Applied Basic Research Fund of Guangdong Province (No. 2019A1515011682), and Guangzhou Regenerative Medicine and Guangdong Laboratory of Health (2019GZR110406004).

\section{Footnote}

Reporting Checklist: The authors have completed the STROBE reporting checklist. Available at http://dx.doi. org/10.21037/jtd-20-3422

Data Sharing Statement: Available at http://dx.doi. org/10.21037/jtd-20-3422 
Conflicts of Interest: All authors have completed the ICMJE uniform disclosure form (available at http://dx.doi. org/10.21037/jtd-20-3422). The authors have no conflicts of interest to declare.

Ethical Statement: The authors are accountable for all aspects of the work in ensuring that questions related to the accuracy or integrity of any part of the work are appropriately investigated and resolved. This study was approved by the human investigation committee of Sun Yatsen Memorial Hospital and was conformed to the provisions of the Declaration of Helsinki (as revised in 2013). Because of the retrospective nature of the research, the requirement for informed consent was waived.

Open Access Statement: This is an Open Access article distributed in accordance with the Creative Commons Attribution-NonCommercial-NoDerivs 4.0 International License (CC BY-NC-ND 4.0), which permits the noncommercial replication and distribution of the article with the strict proviso that no changes or edits are made and the original work is properly cited (including links to both the formal publication through the relevant DOI and the license). See: https://creativecommons.org/licenses/by-nc-nd/4.0/.

\section{References}

1. Howard DP, Banerjee A, Fairhead JF, et al. Populationbased study of incidence and outcome of acute aortic dissection and premorbid risk factor control: 10-year results from the oxford vascular study. Circulation 2013;127:2031-7.

2. Clouse WD, Hallett JW Jr, Schaff HV, et al. Acute aortic dissection: Population-based incidence compared with degenerative aortic aneurysm rupture. Mayo Clin proc 2004;79:176-80.

3. Okita Y. Surgery for acute proximal non-communicating aortic dissection without intimal tears (intramural hematoma). Ann Cardiothorac Surg 2019;8:570-3.

4. Saremi F, Hassani C, Lin LM, et al. Image predictors of treatment outcome after thoracic aortic dissection repair. Radiographics 2018;38:1949-72.

5. Chiappini B, Schepens M, Tan E, et al. Early and late outcomes of acute type A aortic dissection: Analysis of risk factors in 487 consecutive patients. Eur Heart J 2005;26:180-6.

6. Pape LA, Tsai TT, Isselbacher EM, et al. Aortic diameter $>$ or $=5.5 \mathrm{~cm}$ is not a good predictor of type A aortic dissection: Observations from the international registry of acute aortic dissection (IRAD). Circulation 2007;116:1120-7.

7. Ma GG, Hao GW, Lai H, et al. Initial clinical impact of inhaled nitric oxide therapy for refractory hypoxemia following type A acute aortic dissection surgery. J Thorac Dis 2019;11:495-504.

8. Landenhed M, Engstrom G, Gottsater A, et al. Risk profiles for aortic dissection and ruptured or surgically treated aneurysms: A prospective cohort study. J Am Heart Assoc 2015;4:e001513.

9. Mussa FF, Horton JD, Moridzadeh R, et al. Acute aortic dissection and intramural hematoma: A systematic review. JAMA 2016;316:754-63.

10. Wu J, Qiu J, Jiang W, et al. Development and validation of a nomogram predicting the probability of type A aortic dissection at a diameter below $55 \mathrm{~mm}$ : A retrospective cohort study. Int J Surg 2018;60:266-72.

11. Dong N, Piao H, Li B, et al. Poor management of hypertension is an important precipitating factor for the development of acute aortic dissection. J Clin Hypertens (Greenwich) 2019;21:804-12.

12. Kim JB, Kim K, Lindsay ME, et al. Risk of rupture or dissection in descending thoracic aortic aneurysm. Circulation 2015;132:1620-9.

13. Brownstein AJ, Bin Mahmood SU, Saeyeldin A, et al. Simple renal cysts and bovine aortic arch: Markers for aortic disease. Open Heart 2019;6:e000862.

14. Wang Y, Gao P, Du J. Response by wang et al. to letter regarding article, "magnitude of soluble st 2 as a novel biomarker for acute aortic dissection". Circulation 2018;138:1281-2.

15. Zhang L, Xu XM. Letter by zhang and xu regarding article, "magnitude of soluble st 2 as a novel biomarker for acute aortic dissection". Circulation 2018;138:1280.

16. Wang Y, Tan X, Gao H, et al. Magnitude of soluble st2 as a novel biomarker for acute aortic dissection. Circulation 2018;137:259-69.

17. Villahoz S, Yunes-Leites PS, Mendez-Barbero N, et al. Conditional deletion of rcan1 predisposes to hypertensionmediated intramural hematoma and subsequent aneurysm and aortic rupture. Nat Commun 2018;9:4795.

18. Nogi M, Satoh K, Sunamura S, et al. Small GTP-binding protein GDP dissociation stimulator prevents thoracic aortic aneurysm formation and rupture by phenotypic preservation of aortic smooth muscle cells. Circulation 2018;138:2413-33. 
19. Clément M, Chappell J, Raffort J, et al. Vascular smooth muscle cell plasticity and autophagy in dissecting aortic aneurysms. Arterioscler Thromb Vasc Biol 2019;39:1149-59.

20. Cifani N, Proietta M, Taurino M, et al. Monocyte subsets, Stanford-A acute aortic dissection, and carotid artery stenosis: New evidences. J Immunol Res 2019;2019:9782594.

21. Sidloff D, Choke E, Stather P, et al. Mortality from thoracic aortic diseases and associations with cardiovascular risk factors. Circulation 2014;130:2287-94.

22. Ziganshin BA, Theodoropoulos P, Salloum MN, et al. Simple renal cysts as markers of thoracic aortic disease. J Am Heart Assoc 2016;5:e002248.

23. Kim EK, Choi ER, Song BG, et al. Presence of simple renal cysts is associated with increased risk of aortic dissection: A common manifestation of connective tissue degeneration? Heart 2011;97:55-9.

24. Takagi H, Umemoto T, Group A. Simple renal cyst and abdominal aortic aneurysm. J Vasc Surg 2016;63:254259.e1.

25. Lempel JK, Frazier AA, Jeudy J, et al. Aortic arch dissection: A controversy of classification. Radiology 2014;271:848-55.

26. Barbetseas J, Alexopoulos N, Brili S, et al. Atherosclerosis of the aorta in patients with acute thoracic aortic dissection. Circ J 2008;72:1773-6.

27. Svensson LG, Kouchoukos NT, Miller DC, et al. Expert consensus document on the treatment of descending thoracic aortic disease using endovascular stent-grafts. Ann Thorac Surg 2008;85:S1-41.

28. Sueyoshi E, Sakamoto I, Hayashi K, et al. Growth

Cite this article as: Bao J, Zheng S, Huang C, Tao J, Tang Y, Sun R, Guo Q, Wang J, Zhang Y. Association of renal cyst and type A acute aortic dissection with hypertension. J Thorac Dis 2020;12(12):7374-7386. doi: 10.21037/jtd-20-3422 rate of aortic diameter in patients with type B aortic dissection during the chronic phase. Circulation 2004;110:II256-61.

29. Juvonen T, Ergin MA, Galla JD, et al. Prospective study of the natural history of thoracic aortic aneurysms. Ann Thorac Surg 1997;63:1533-45.

30. Lederle FA, Johnson GR, Wilson SE, et al. Rupture rate of large abdominal aortic aneurysms in patients refusing or unfit for elective repair. JAMA 2002;287:2968-72.

31. Evangelista A, Salas A, Ribera A, et al. Long-term outcome of aortic dissection with patent false lumen: Predictive role of entry tear size and location. Circulation 2012;125:3133-41.

32. Lu N, Hu P, Wang J, et al. Simple renal cysts are associated with 24-month prognosis of patients with type $\mathrm{B}$ aortic dissection and hypertension. Can J Cardiol 2019;35:1499-504.

33. Immer FF, Krahenbuhl E, Hagen U, et al. Large area of the false lumen favors secondary dilatation of the aorta after acute type A aortic dissection. Circulation 2005;112:I249-52.

34. Vilacosta I, San Roman JA. Acute aortic syndrome. Heart 2001;85:365-8.

35. Harris KM, Braverman AC, Eagle KA, et al. Acute aortic intramural hematoma: An analysis from the international registry of acute aortic dissection. Circulation 2012;126:S91-6.

36. Tsai TT, Nienaber CA, Eagle KA. Acute aortic syndromes. Circulation 2005;112:3802-13.

(English Language Editor: J. Gray) 

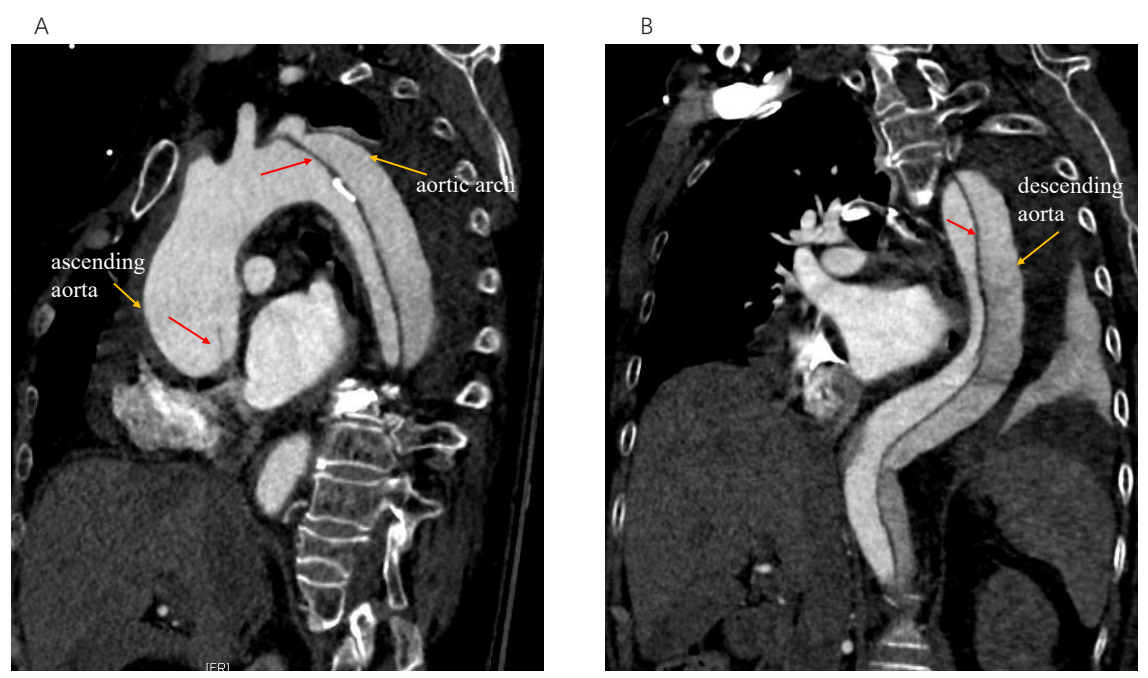

Figure S1 Computed tomography (CT) imaging of Stanford A aortic dissection. The red arrow is aortic dissection, and the yellow arrow is the vessel name. (A) There has aortic dissection in ascending aorta and aortic arch; (B) there has aortic dissection in descending aorta. 